\title{
Improved Dirac quantization of a free particle
}

\author{
Soon-Tae Hong*, Won Tae Kim ${ }^{\dagger}$ and Young-Jai Park ${ }^{\ddagger}$ \\ Department of Physics and Basic Science Research Institute, \\ Sogang University, C.P.O. Box 1142, Seoul 100-611, Korea
}

(May 1, 2019)

\begin{abstract}
In the framework of Dirac quantization with second class constraints, a free particle moving on the surface of a $(d-1)$-dimensional sphere has an ambiguity in the energy spectrum due to the arbitrary shift of canonical momenta. We explicitly show that this spectrum obtained by the Dirac method can be consistent with the result of the Batalin-Fradkin-Tyutin formalism, which is an improved Dirac method, at the level of the first-class constraint by fixing the ambiguity, and discuss its physical consequences.
\end{abstract}

PACS: 12.39.Dc, 11.10.Ef, 14.20.-c

Keywords: free particle on a sphere, Dirac quantization, BFT formalism

*electronic address:sthong@ccs.sogang.ac.kr

†electronic address:wtkim@ccs.sogang.ac.kr

‡electronic address:yjpark@ccs.sogang.ac.kr 
It is well known that the canonical quantization of a free point particle in a curved space is a long-standing and controversial problem in quantum mechanics [1 1 . Indeed, for such a system the classical-quantum correspondence does not uniquely define a Hamiltonian operator and this ambiguity affects the energy spectrum of the physical system.

On the other hand, in order to quantize the physical systems subjected to the constraints, the Dirac quantization scheme [5] has been used widely. However, whenever we adopt the Dirac method, we frequently meet the problem of the operator ordering ambiguity. In order to avoid this problem, Batalin, Fradkin, and Tyutin (BFT) developed a method [6] converting the second-class constraints into first-class ones, which instead of configuration space restricts the quantum-mechanical Hilbert space. Then, the operators representing the first-class constraints are generators of gauge transformations, and the physical states are all found by going into the gauge invariant subspace of the Hilbert space. Recently, this BFT formalism has been applied to several interesting models [7]. Very recently, the SU(2) Skyrme model has been studied in the context of the BFT formalism [8,9].

In this Letter, we will perform a Hamiltonian quantization of a free particle moving on the surface of a $(d-1)$-dimensional sphere by exactly identifying the ambiguity of the energy spectrum. We show how this ambiguous spectrum obtained by the Dirac method can be consistent with the result of the BFT formalism, which is an improved version of Dirac method. Firstly, the Dirac bracket scheme will be applied to a free particle constrained on a $(d-1)$-dimensional sphere. The adjustable parameter will be introduced to define the generalized momenta without any loss of generality, which yields an ambiguous energy spectrum. Next, we will apply the BFT method to this model to obtain the energy spectrum including the Weyl ordering correction. Then, we will show that by fixing this free parameter the energy eigenvalues obtained by the Dirac method are consistent with the result of the BFT formalism. Finally, we will construct the BRST invariant gauge fixed Lagrangian [10] as well as the effective Lagrangian corresponding to the first-class Hamiltonian in the Batalin, Fradkin and Vilkovisky (BFV) scheme [11 13].

Now we start with the following Lagrangian describing a free particle with a unit mass 
on a $(d-1)$-dimensional sphere of unit radius embedded in a $d$-dimensional Cartesian space with coordinates $q_{i}(i=1,2, . ., d)$ :

$$
L=\frac{1}{2} \dot{q}_{i} \dot{q}_{i}
$$

Introducing the canonical momenta $\pi_{i}=\dot{q}_{i}$ conjugate to the coordinates $q_{i}$ one can then obtain the canonical Hamiltonian

$$
H=\frac{1}{2} \pi_{i} \pi_{i}
$$

On the other hand, we have the following second-class constraints:

$$
\Omega_{1}=q_{i} q_{i}-1 \approx 0, \quad \Omega_{2}=q_{i} \pi_{i} \approx 0
$$

to yield the Poisson algebra $\Delta_{a b}=\left\{\Omega_{a}, \Omega_{b}\right\}=2 \epsilon^{a b} q_{i} q_{i}$ with $\epsilon^{12}=-\epsilon^{21}=1$. Here one notes that, due to the commutator $\left\{\pi_{i}, \Omega_{1}\right\}=-2 q_{i}$, one can easily obtain the algebraic relation $\left\{\Omega_{1}, H\right\}=2 \Omega_{2}$. Using the Dirac brackets [5] defined by $\{A, B\}_{D}=$ $\{A, B\}-\left\{A, \Omega_{a}\right\} \Delta^{a b}\left\{\Omega_{b}, B\right\}$ with $\Delta^{a b}$ being the inverse of $\Delta_{a b}$ and performing the canonical quantization scheme $\{A, B\}_{D} \rightarrow \frac{1}{i}\left[A_{o p}, B_{o p}\right]$, one can obtain the operator commutators

$$
\begin{aligned}
{\left[q_{i}, q_{j}\right] } & =0 \\
{\left[q_{i}, \pi_{j}\right] } & =i\left(\delta_{i j}-\frac{q_{i} q_{j}}{q_{k} q_{k}}\right), \\
{\left[\pi_{i}, \pi_{j}\right] } & =\frac{i}{q_{k} q_{k}}\left(q_{j} \pi_{i}-q_{i} \pi_{j}\right)
\end{aligned}
$$

with $\pi_{i}=-i\left(\delta_{i j}-\frac{q_{i} q_{j}}{q_{k} q_{k}}\right) \partial_{j}$.

Then we observe that without any loss of generality the generalized momenta $\Pi_{i}$ fulfilling the structure of the commutators (3) are given by $\Pi_{i}=-i\left(\delta_{i j}-\frac{q_{i} q_{j}}{q_{k} q_{k}}\right) \partial_{j}-\frac{i c q_{i}}{q_{k} q_{k}}$ with an arbitrary parameter $c$ to be fixed later [9,16]. In Ref. [14, 15] the authors did not include the last term so that one cannot clarify the relations between the BFT scheme and the Dirac bracket one. On the other hand, the energy spectrum of the free particle can be obtained in the Weyl ordering scheme [17] where the Hamiltonian (2) is modified into the symmetric form

$$
H_{N}=\frac{1}{2} \Pi_{i}^{N} \Pi_{i}^{N},
$$


where

$$
\Pi_{i}^{N}=-\frac{i}{2}\left[\left(\delta_{i j}-\frac{q_{i} q_{j}}{q_{k} q_{k}}\right) \partial_{j}+\partial_{j}\left(\delta_{i j}-\frac{q_{i} q_{j}}{q_{k} q_{k}}\right)+\frac{2 c q_{i}}{q_{k} q_{k}}\right] .
$$

After some algebra, one can obtain the Weyl ordered $\Pi_{i}^{N} \Pi_{i}^{N}$ as follows:

$$
\Pi_{i}^{N} \Pi_{i}^{N}=-\partial_{i} \partial_{i}+\frac{(d-1) q_{i}}{q_{k} q_{k}} \partial_{i}+\frac{q_{i} q_{j}}{q_{k} q_{k}} \partial_{i} \partial_{j}+\frac{1}{q_{k} q_{k}}\left[\frac{(d-1)^{2}}{4}-c^{2}\right],
$$

which yields the modified quantum energy spectrum as

$$
\left\langle H_{N}\right\rangle=\frac{1}{2}\left[l(l+d-2)+\frac{(d-1)^{2}}{4}-c^{2}\right] .
$$

Here the first three terms in Eq. (4) are nothing but the $(d-1)$-sphere Laplacian [18] given in terms of the coordinates and their derivatives to yield the eigenvalues $l(l+d-2)$. Note that due to the ambiguity of the arbitrary value $c$, we could adjust any energy spectrum obtained by various approaches [2, [] to give the proper spectrum. In fact one cannot fix uniquely the energy spectrum only by using the Dirac method.

Next, following the Abelian BFT formalism [6 8] which systematically converts the second-class constraints into the first-class ones, we introduce two auxiliary fields $\Phi^{a}$ corresponding to $\Omega_{a}$ with the Poisson brackets $\left\{\Phi^{a}, \Phi^{b}\right\}=\omega^{a b}$. The first-class constraints $\tilde{\Omega}_{a}$ are then constructed as a power series of the auxiliary fields:

$$
\tilde{\Omega}_{a}=\sum_{n=0}^{\infty} \Omega_{a}^{(n)}, \quad \Omega_{a}^{(0)}=\Omega_{a}
$$

where $\Omega_{a}^{(n)}$ are polynomials in the auxiliary fields $\Phi^{a}$ of degree $n$, to be determined by the requirement that the first-class constraints $\tilde{\Omega}_{a}$ satisfy an Abelian algebra $\left\{\tilde{\Omega}_{a}, \tilde{\Omega}_{b}\right\}=0$. Following the standard iterating procedure with the choice of $\omega^{a b}=\epsilon^{a b}$ [6, 7], one can obtain the first-class constraints

$$
\tilde{\Omega}_{1}=\Omega_{1}+2 \Phi^{1}, \tilde{\Omega}_{2}=\Omega_{2}-q_{i} q_{i} \Phi^{2}
$$

which yield the strongly involutive first-class constraint algebra $\left\{\tilde{\Omega}_{a}, \tilde{\Omega}_{b}\right\}=0$.

Now we systematically construct the first-class BFT physical fields $\tilde{\mathcal{F}}=\left(\tilde{q}_{i}, \tilde{\pi}_{i}\right)$ in the extended phase space corresponding to the original fields $\mathcal{F}=\left(q_{i}, \pi_{i}\right)$, which are obtained 
as a power series in the auxiliary fields $\Phi^{a}$ by demanding that they are strongly involutive: $\left\{\tilde{\Omega}_{a}, \tilde{\mathcal{F}}\right\}=0$. In general the first-class-fields satisfying the boundary conditions $\tilde{\mathcal{F}}[\mathcal{F} ; 0]=\mathcal{F}$ can be found as

$$
\tilde{\mathcal{F}}[\mathcal{F} ; \Phi]=\mathcal{F}+\sum_{n=1}^{\infty} \tilde{\mathcal{F}}^{(n)}, \quad \tilde{\mathcal{F}}^{(n)} \sim(\Phi)^{n}
$$

where the $(n+1)$-th order iteration terms are given by the formula

$$
\tilde{\mathcal{F}}^{(n+1)}=-\frac{1}{n+1} \Phi^{a} \omega_{a b} X^{b c} G_{c}^{(n)}
$$

with

$$
G_{a}^{(n)}=\sum_{m=0}^{n}\left\{\Omega_{a}^{(n-m)}, \tilde{\mathcal{F}}^{(m)}\right\}_{(\mathcal{F})}+\sum_{m=0}^{n-2}\left\{\Omega_{a}^{(n-m)}, \tilde{\mathcal{F}}^{(m+2)}\right\}_{(\Phi)}+\left\{\Omega_{a}^{(n+1)}, \tilde{\mathcal{F}}^{(1)}\right\}_{(\Phi)}
$$

After some algebra, we obtain the first-class physical fields,

$$
\begin{aligned}
& \tilde{q}_{i}=q_{i}\left[1-\sum_{n=1}^{\infty} \frac{(-1)^{n}(2 n-3) ! !}{n !} \frac{\left(\Phi^{1}\right)^{n}}{\left(q_{k} q_{k}\right)^{n}}\right] \\
& \tilde{\pi}_{i}=\left(\pi_{i}-q_{i} \Phi^{2}\right)\left[1+\sum_{n=1}^{\infty} \frac{(-1)^{n}(2 n-1) ! !}{n !} \frac{\left(\Phi^{1}\right)^{n}}{\left(q_{k} q_{k}\right)^{n}}\right]
\end{aligned}
$$

with $(-1) ! !=1$.

Then, using the novel property [19] that any functional $\mathcal{K}(\tilde{\mathcal{F}})$ of the first-class fields $\tilde{\mathcal{F}}$ will also be first-class, i.e., $\tilde{\mathcal{K}}(\mathcal{F} ; \Phi)=\mathcal{K}(\tilde{\mathcal{F}})$, we can directly construct the first-class Hamiltonian in terms of the above BFT physical variables as follows

$$
\tilde{H}=\frac{1}{2} \tilde{\pi}_{i} \tilde{\pi}_{i}
$$

omitting infinitely iterated standard procedure [9].

As a result, the corresponding first-class Hamiltonian with the original fields and auxiliary fields is given by

$$
\tilde{H}=\frac{1}{2}\left(\pi_{i}-q_{i} \Phi^{2}\right)\left(\pi_{i}-q_{i} \Phi^{2}\right) \frac{q_{j} q_{j}}{q_{j} q_{j}+2 \Phi^{1}},
$$

which is also strongly involutive with the first-class constraints $\left\{\tilde{\Omega}_{a}, \tilde{H}\right\}=0$. However, with the Hamiltonian (9), one cannot naturally generate the first-class Gauss' law constraint 
from the time evolution of the primary constraint $\tilde{\Omega}_{1}$. By introducing an additional term proportional to the first-class constraints $\tilde{\Omega}_{2}$ into $\tilde{H}$, we obtain an equivalent first-class Hamiltonian

$$
\tilde{H}^{\prime}=\tilde{H}+\Phi^{2} \tilde{\Omega}_{2},
$$

which naturally generates the Gauss' law constraint, $\left\{\tilde{\Omega}_{1}, \tilde{H}^{\prime}\right\}=2 \tilde{\Omega}_{2}$ and $\left\{\tilde{\Omega}_{2}, \tilde{H}^{\prime}\right\}=0$. Here one notes that $\tilde{H}$ and $\tilde{H}^{\prime}$ act on physical states in the same way since such states are annihilated by the first-class constraints. Similarly, the equations of motion for observables are also unaffected by this difference. Furthermore, if we take the limit $\Phi^{a} \rightarrow 0$, then our first-class system exactly returns to the original second-class one.

We are now ready to obtain the energy spectrum of the extended phase space. The fundamental idea consists in imposing quantum mechanically the first-class constraints as operator condition on the state as a way to obtain the physical subspace, i.e., $\tilde{\Omega}_{a} \mid$ phys $>=0$ where we used the symmetrized operators as $\tilde{\Omega}_{1}=q_{i} q_{i}+2 \Phi^{1}$ and $\tilde{\Omega}_{2}=\left(q_{i} \pi_{i}\right)_{\mathrm{sym}}-q_{i} q_{i} \Phi^{2}$. Then, after the symmetrization procedure [8], the first-class Hamiltonian yields the energy spectrum with the Weyl ordering correction

$$
\left\langle\tilde{H}_{N}^{\prime}\right\rangle=\frac{1}{2}\left[l(l+d-2)+\frac{d(d-3)}{4}\right] .
$$

This result obtained through the Abelian BFT analysis is well in agreement with the energy level spacings due to the angular contribution of the hydrogen atom because there is no additional constant parameter in the energy eigenvalues for the case of $d=3$. Furthermore, our result well describes the spectrum of $\mathrm{SU}(2)$ Skyrmion model corresponding to the $d=4$ case [8.9]. Note that, however, the recent result obtained from the unusual non-Abelian BFT scheme [14] can not describe the correct situation for $d=3$ case. The reason is that this can not naturally generate the Gauss' law constraint, and does not recover the original second-class constraint structure in the limit of $\Phi^{a} \rightarrow 0$.

Now, in order for the Dirac bracket scheme to be consistent with the BFT one, the 
adjustable parameter $c$ in Eq. (5) should be fixed with the values

$$
c= \pm \frac{\sqrt{d+1}}{2}
$$

Then, this fixed parameter $c$ relates the Dirac bracket scheme to the BFT one to yield the desired quantization in the model of the free particle on $(d-1)$-sphere so that one can achieve the unification of these two formalisms.

Next, let us consider the partition function of the model in order to present the Lagrangian corresponding to the first-class Hamiltonian $\tilde{H}^{\prime}$ in the canonical Hamiltonian formalism. First of all, let us identify the auxiliary fields $\Phi^{a}$ with a canonical conjugate pair $\left(\theta, \pi_{\theta}\right)$, i.e., $\Phi^{a}=\left(\theta, \pi_{\theta}\right)$ which satisfy $\left\{\Phi^{a}, \Phi^{b}\right\}=\omega^{a b}$ with $\omega^{a b}=\epsilon^{a b}$. Then, the starting partition function in the phase space is given by the Faddeev-Senjanovic formula [20] as follows

$$
Z=N \int \mathcal{D} q_{i} \mathcal{D} \pi_{i} \mathcal{D} \theta \mathcal{D} \pi_{\theta} \prod_{a, b=1}^{2} \delta\left(\tilde{\Omega}_{a}\right) \delta\left(\Gamma_{b}\right) \operatorname{det}|M| \exp i \int \mathrm{d} t\left(\pi_{i} \dot{q}_{i}+\pi_{\theta} \dot{\theta}-\tilde{H}^{\prime}\right)
$$

where the gauge fixing conditions $\Gamma_{i}$ are chosen so that the determinant occurring in the functional measure is nonvanishing, and $M=\left\{\tilde{\Omega}_{a}, \Gamma_{b}\right\}$.

Now, exponentiating the delta function $\delta\left(\tilde{\Omega}_{2}\right)$ as $\delta\left(\tilde{\Omega}_{2}\right)=\int \mathcal{D} \xi e^{i \int \mathrm{d} t \xi \tilde{\Omega}_{2}}$ and performing the integration over $\pi_{\theta}$, we obtain

$$
\begin{aligned}
& Z=N \int \mathcal{D} q_{i} \mathcal{D} \pi_{i} \mathcal{D} \theta \mathcal{D} \xi \delta\left(q_{i} q_{i}-1+2 \theta\right) \prod_{a=1}^{2} \delta\left(\Gamma_{a}\right) \operatorname{det}|M| e^{i \int \mathrm{d} t L} \\
& L=-\frac{1}{2} q_{i} q_{i} \pi_{j} \pi_{j}+\left(\dot{q}_{i}-\xi q_{i}\right) \pi_{i}-\frac{1}{2\left(q_{k} q_{k}\right)^{2}}\left(\dot{\theta}+\xi q_{i} q_{i}\right)^{2} .
\end{aligned}
$$

After integrating out the momenta $\pi_{i}$ and auxiliary field $\xi$, the partition function is given as follows

$$
\begin{aligned}
& Z=N \int \mathcal{D} q_{i} \mathcal{D} \theta \delta\left(q_{i} q_{i}-1+2 \theta\right) \prod_{a=1}^{2} \delta\left(\Gamma_{a}\right) \operatorname{det}|M| e^{i \int \mathrm{d} t L} \\
& L=\frac{1}{2 q_{k} q_{k}} \dot{q}_{i} \dot{q}_{i}-\frac{1}{2\left(q_{k} q_{k}\right)^{2}} \dot{\theta}^{2} .
\end{aligned}
$$

As a result, we have obtained the desired Lagrangian (12) corresponding to the first-class Hamiltonian (10). Here one notes that the Lagrangian (12) can be re-shuffled to yield the gauge invariant action of the form 


$$
\begin{aligned}
S & =\int \mathrm{d} t\left(\frac{1}{2} \dot{q}_{i} \dot{q}_{i}\right)+S_{W Z} \\
S_{W Z} & =\int \mathrm{d} t\left[\frac{1}{q_{k} q_{k}} \dot{q}_{i} \dot{q}_{i} \theta-\frac{1}{2\left(q_{k} q_{k}\right)^{2}} \dot{\theta}^{2}\right],
\end{aligned}
$$

where $S_{W Z}$ is the new type of the Wess-Zumino term restoring the gauge symmetry under the transformation: $\delta q_{i}=q_{i} \epsilon, \delta \theta=-q_{i} q_{i} \epsilon$ where $\epsilon$ is a local gauge parameter. Here one notes that this form of symmetry transformation is exactly the same as that obtained when we consider the effective first-class constraints (7) as the symmetry generators in the Hamiltonian formalism.

Moreover the corresponding partition function (11) can be rewritten simply in terms of the first-class physical fields (8)

$$
\tilde{Z}=N \int \mathcal{D} \tilde{q}_{i} \delta\left(\tilde{q}_{j} \tilde{q}_{j}-1\right) \prod_{a=1}^{2} \delta\left(\Gamma_{a}\right) \operatorname{det}|M| \exp i \int \mathrm{d} t\left(\frac{1}{2} \dot{\tilde{q}}_{i} \dot{\tilde{q}}_{i}\right)
$$

where $\tilde{L}$ is form invariant Lagrangian of Eq. (11).

Now, in order to obtain the BRST invariant gauge fixed Lagrangian, we introduce two canonical sets of ghosts and anti-ghosts together with auxiliary fields in the framework of the BFV formalism [11]13, which is applicable to theories with the first-class constraints: $\left(\mathcal{C}^{a}, \overline{\mathcal{P}}_{a}\right),\left(\mathcal{P}^{a}, \overline{\mathcal{C}}_{a}\right),\left(N^{a}, B_{a}\right),(a=1,2)$ which satisfy the super-Poisson algebra $\left\{\mathcal{C}^{a}, \overline{\mathcal{P}}_{b}\right\}=$ $\left\{\mathcal{P}^{a}, \overline{\mathcal{C}_{b}}\right\}=\left\{N^{a}, B_{b}\right\}=\delta_{b}^{a}$. The super-Poisson bracket is defined as $\{A, B\}=\left.\left.\frac{\delta A}{\delta q}\right|_{r} \frac{\delta B}{\delta p}\right|_{l}-$ $\left.\left.(-1)^{\eta_{A} \eta_{B}} \frac{\delta B}{\delta q}\right|_{r} \frac{\delta A}{\delta p}\right|_{l}$ where $\eta_{A}$ denotes the number of fermions called ghost number in $A$ and the subscript $r$ and $l$ right and left derivatives. In the model for the free particle on a $(d-$ 1)-dimensional sphere, the nilpotent $\operatorname{BRST}$ charge $Q$, the fermionic gauge fixing function $\Psi$ and the BRST invariant minimal Hamiltonian $H_{m}$ are given by

$$
Q=\mathcal{C}^{a} \tilde{\Omega}_{a}+\mathcal{P}^{a} B_{a}, \quad \Psi=\overline{\mathcal{C}}_{a} \chi^{a}+\overline{\mathcal{P}}_{a} N^{a}, \quad H_{m}=\tilde{H}^{\prime}-2 \mathcal{C}^{1} \overline{\mathcal{P}}_{2}
$$

which satisfy the relations $\left\{Q, H_{m}\right\}=0, Q^{2}=\{Q, Q\}=0$, and $\{\{\Psi, Q\}, Q\}=0$. The effective quantum Lagrangian is then described as

$$
L_{e f f}=\pi_{i} \dot{q}_{i}+\pi_{\theta} \dot{\theta}+B_{2} \dot{N}^{2}+\overline{\mathcal{P}}_{a} \dot{\mathcal{C}}^{a}+\overline{\mathcal{C}}_{2} \dot{\mathcal{P}}^{2}-H_{t o t}
$$


with $H_{t o t}=H_{m}-\{Q, \Psi\}$. Here $B_{1} \dot{N}^{1}+\overline{\mathcal{C}}_{1} \dot{\mathcal{P}}^{1}=\left\{Q, \overline{\mathcal{C}}_{1} \dot{N}^{1}\right\}$ terms are suppressed by replacing $\chi^{1}$ with $\chi^{1}+\dot{N}^{1}$.

Now we choose the unitary gauge $\chi^{1}=\Omega_{1}, \chi^{2}=\Omega_{2}$ and perform the path integration over the fields $B_{1}, N^{1}, \overline{\mathcal{C}}_{1}, \mathcal{P}^{1}, \overline{\mathcal{P}}_{1}$ and $\mathcal{C}^{1}$, by using the equations of motion, to yield the effective Lagrangian of the form

$$
\begin{aligned}
L_{e f f}= & \pi_{i} \dot{q}_{i}+\pi_{\theta} \dot{\theta}+B \dot{N}+\overline{\mathcal{P}} \dot{\mathcal{C}}+\overline{\mathcal{C}} \dot{\mathcal{P}} \\
& -\frac{1}{2}\left(\pi_{i}-q_{i} \pi_{\theta}\right)\left(\pi_{i}-q_{i} \pi_{\theta}\right) \frac{q_{j} q_{j}}{q_{j} q_{j}+2 \theta}-\pi_{\theta} \tilde{\Omega}_{2} \\
& +2 q_{i} q_{i} \pi_{\theta} \overline{\mathcal{C}} \mathcal{C}+\tilde{\Omega}_{2} N+B \Omega_{2}+\overline{\mathcal{P}} \mathcal{P}
\end{aligned}
$$

with redefinitions: $N \equiv N^{2}, B \equiv B_{2}, \overline{\mathcal{C}} \equiv \overline{\mathcal{C}}_{2}, \mathcal{C} \equiv \mathcal{C}^{2}, \overline{\mathcal{P}} \equiv \overline{\mathcal{P}}_{2}, \mathcal{P} \equiv \mathcal{P}_{2}$

Then, using the variations with respect to $\pi_{i}, \pi_{\theta}, \mathcal{P}$ and $\overline{\mathcal{P}}$ and identifying $N$ with $N=-B+\frac{\dot{\theta}}{q_{k} q_{k}}$, we obtain the desired effective Lagrangian

$$
L_{e f f}=\frac{1}{2 q_{k} q_{k}} \dot{q}_{i} \dot{q}_{i}-\frac{1}{2\left(q_{k} q_{k}\right)^{2}} \dot{\theta}^{2}-\frac{1}{2}\left(q_{k} q_{k}\right)^{2}(B+2 \overline{\mathcal{C}} \mathcal{C})^{2}-\frac{\dot{\theta} \dot{B}}{q_{k} q_{k}}+\dot{\overline{\mathcal{C}}} \dot{\mathcal{C}},
$$

which is invariant under the BRST transformation

$$
\begin{aligned}
\delta_{B} q_{i} & =\lambda q_{i} \mathcal{C}, \quad \delta_{B} \theta=-\lambda q_{i} q_{i} \mathcal{C}, \\
\delta_{B} \overline{\mathcal{C}} & =-\lambda B, \quad \delta_{B} \mathcal{C}=\delta_{B} B=0 .
\end{aligned}
$$

Here one notes that the above BRST transformation including the rules for the (anti)ghost fields is just the generalization of the previous one $\delta q_{i}=q_{i} \epsilon, \delta \theta=-q_{i} q_{i} \epsilon$.

In summary, we have clarified the relation between the Dirac bracket scheme with the second-class constraints and the BFT method at the level of the first-class constraint, which has been obscure and unsettled, in the free particle on a $(d-1)$-dimensional sphere. In this approach we have introduced the generalized momentum operators including the free parameter, which is fixed to yield the consistency between these two formalisms. We have shown that one could see the effects of the Weyl ordering correction in the energy spectrum. Note that the energy spectrum is remarkably reproduced for $d=3$ and $d=4$ which correspond to the explicit physical phenomena, three-dimensional rotator and SU(2) Skyrmion, respectively. 


\section{Acknowledgments}

The present work was supported by the Sogang University Research Grants in 1999 and by the Ministry of Education, BK21 Project No. D-1099, 1999. 


\section{REFERENCES}

[1] B. Podolsky, Phys. Rev. 32, 812 (1928); B. De Witt, Phys. Rev. 85, 653 (1952); L. Landau and E.M. Lifshitz, Quantum Mechanics, (Pergamon, New York, 1965).

[2] B. De Witt, Rev. Mod. Phys. 29, 377 (1957); K. S. Cheng, J. Math. Phys. 13, 1723 (1972); H. Dekker, Physica 103A, 586 (1980).

[3] M. S. Marinov, Phys. Rep. C60, 25 (1980); N. K. Falck and A. C. Hirshfield, Eur. J. Phys. 4, 5 (1983); T. Homma, T. Inamoto, and T. Miyazaki, Phys. Rev. D42, 2049 (1990); A. Foerster, H. O. Girotti, and P. S. Kuhn, Phys. Lett. 195A, 301 (1994).

[4] P. Dita, Phys. Rev. A56, 2574 (1997); quant-ph/9809083; H. Kleinert and S. V. Shabanov, Phys. Lett. A232, 327 (1997); J.R. Klauder and S.V. Shabanov, Nucl. Phys. B511, 713 (1998); E. Abdalla and R. Banerjee, quant-ph/9803021.

[5] P. A. M. Dirac, Lectures in Quantum Mechanics (Yeshiva University, New York, 1964).

[6] I. A. Batalin, and E. S. Fradkin, Phys. Lett. B180, 157 (1986); Nucl. Phys. B279, 514 (1987); I. A. Batalin, and I. V. Tyutin, Int. J. Mod. Phys. A6, 3255 (1991).

[7] R. Banerjee, Phys. Rev. D48, R5467 (1993); W. T. Kim, and Y.-J. Park, Phys. Lett. B336, 376 (1994); Y.-W. Kim and Y.-J. Park, Mod. Phys. Lett. A13, 1201 (1998); Y.-W. Kim, W.T. Kim, and Y.-J. Park, J. Phys. A32, 2461 (1999); M. Fleck and H. O. Girotti, Int. J. Mod. Phys. A14, 4287 (1999).

[8] W. Oliveira, and J. A. Neto, Int. J. Mod. Phys. A12, 4895 (1997).

[9] S.-T. Hong, Y.-W. Kim, and Y.-J. Park, Phys. Rev. D59, 114026 (1999); S.-T. Hong, Y.-W. Kim, and Y.-J. Park, Mod. Phys. Lett. A15, 55 (2000).

[10] C. Becci, A. Rouet, and R. Stora, Ann. Phys. 98, 287 (1976); I. V. Tyutin, Levedev report $39(1975)$.

[11] E.S. Fradkin, G.A. Vilkovisky, Phys. Lett. B55, 224 (1975); M. Henneaux, Phys. Rep. 
C126, 1 (1985).

[12] T. Fujiwara, Y. Igarashi, J. Kubo, Nucl. Phys. B341, 695 (1990) ; Y.-W. Kim, S.-K. Kim, W. T. Kim, Y.-J. Park, K. Y. Kim, Y. Kim, Phys. Rev. D46, 4574 (1992).

[13] C. Bizdadea, S.O. Saliu, Nucl. Phys. B456, 473 (1995).

[14] J. A. Neto and W. Oliveira, Int. J. Mod. Phys. A14, 3699 (1999).

[15] K. Fujii, and N. Ogawa, Prog. Theor. Phys. Suppl. 109, 1 (1986).

[16] J. A. Neto, J. Phys. G21, 695 (1995).

[17] T. D. Lee, Particle Physics and Introduction to Field Theory (Harwood, New York, 1981).

[18] N. Vilenkin, Special Functions and the Theory of Group Representations, Amer. Math. Soc., Providence, R.I., 1968.

[19] W. T. Kim, Y.-W. Kim, M.-I. Park, Y.-J. Park, and S. J. Yoon, J. Phys. G23, 325 (1997); M.-I. Park, Y.-J. Park, and S.-J. Yoon, J. Phys. G24, 2179 (1998).

[20] L.D. Faddeev, Theor. Math. Phys. 1, 1 (1970); P. Senjanovich, Ann. Phys. (N.Y.) 100, 277 (1976). 